LAWRENCE LIVERMORE NATIONAL LABORATORY
Re-examining the Dissolution of Spent Fuel: A Comparison of Different Methods for Calculating Rates

B.D. Hanson, R.B. Stout

April 9, 2004

Spring Material Research Society Meeting San Francisco, CA

April 12-16, 2004 
This document was prepared as an account of work sponsored by an agency of the United States Government. Neither the United States Government nor the University of California nor any of their employees, makes any warranty, express or implied, or assumes any legal liability or responsibility for the accuracy, completeness, or usefulness of any information, apparatus, product, or process disclosed, or represents that its use would not infringe privately owned rights. Reference herein to any specific commercial product, process, or service by trade name, trademark, manufacturer, or otherwise, does not necessarily constitute or imply its endorsement, recommendation, or favoring by the United States Government or the University of California. The views and opinions of authors expressed herein do not necessarily state or reflect those of the United States Government or the University of California, and shall not be used for advertising or product endorsement purposes. 


\title{
Re-examining the Dissolution of Spent Fuel: A Comparison of Different Methods for Calculating Rates
}

\author{
Brady D. Hanson and Ray B. Stout ${ }^{1}$ \\ Radiochemical Science \& Engineering Group, \\ Pacific Northwest National Laboratory, Richland, WA 99352 \\ ${ }^{1}$ University of California/LLNL, Livermore, CA 94550
}




\title{
Re-examining the Dissolution of Spent Fuel: A Comparison of Different Methods for Calculating Rates
}

\author{
Brady D. Hanson and Ray B. Stout ${ }^{1}$ \\ Radiochemical Science \& Engineering Group, Pacific Northwest National Laboratory, \\ Richland, WA 99352, U.S.A. \\ ${ }^{1}$ University of California/LLNL, Livermore, CA 94550, U.S.A.
}

\begin{abstract}
Dissolution rates for spent fuel have typically been reported in terms of a rate normalized to the surface area of the specimen. Recent evidence has shown that neither the geometric surface area nor that measured with BET accurately predicts the effective surface area of spent fuel. Dissolution rates calculated from results obtained by flowthrough tests were reexamined comparing the cumulative releases and surface area normalized rates. While initial surface area is important for comparison of different rates, it appears that normalizing to the surface area introduces unnecessary uncertainty compared to using cumulative or fractional release rates. Discrepancies in past data analyses are mitigated using this alternative method.
\end{abstract}

\section{INTRODUCTION}

Leaching experiments on $\mathrm{UO}_{2}$ and spent nuclear fuels have been performed to determine the rate of radionuclide release from fuel exposed to water in proposed geologic repositories. Since only the atoms at the surface of the specimen are actually subjected to contact with water, dissolution rates have typically been reported as normalized to the exposed surface area (e.g., mg $\left.\mathrm{m}^{-2} \mathrm{~d}^{-1}\right)$. The exposed surface area is usually determined as the geometric surface area, typically calculated from particle-size distribution measurements and often multiplied by a surface roughness factor [1], or measured using the Brunauer, Emmett, and Teller (BET) [2] method.

Virtually all dissolution tests on $\mathrm{UO}_{2}$ and spent fuel exhibit decreasing rates as time and the extent of reaction increase [3-8]. For static or low-flow tests, such behavior can be explained by the formation of alteration products on the surface of the specimen that limit water contact with the matrix, decrease the sites for $\mathrm{O}_{2}$ reduction [9], or incorporate radionuclides and delay or prevent their release. However, for flowthrough tests where no back reactions, at least for uranium, occur, similar decreases have been observed [1,6-7]. It is often assumed that the early initial releases are due to an oxidized layer of the fuel. Serrano et. al. [8] have shown that this effect appears to be relatively transient and short-lived and does not explain the continuing decrease in reaction rate over time.

Röllin et. al. [5] reported that the decreases were a result of corrosion of the stainless steel frits used to hold the fuel in the column, leading to reduction of radionuclides by iron. While the reductive capacity of iron may be an issue, it does not explain the lack of reproducibility in tests, especially since corrosion of stainless steel under these conditions should be minimal. For 
example, Oversby [10] has stated that there is a difference in dissolution rates for SIMFUEL of up to a factor of 100 for conditions that should be expected to give similar results. Similar discrepancies are often observed for spent fuel and even unirradiated $\mathrm{UO}_{2}$ tests as well. In light of the decrease in rates reported for low $\mathrm{pH}$ conditions [7] and the apparent change in effective surface area, a reexamination of previously reported $[1,6]$ and new flowthrough data was performed.

\section{THEORY}

It has long been believed that the rate of fuel dissolution is directly proportional to the surface area of the specimen exposed to solution. Accurately determining this surface area has been difficult because of the structure of $\mathrm{UO}_{2}$ and the propensity to react grain boundaries. Geometric surface areas may be determined relatively easily and consistently, however, Gray [1] showed that these surface areas do not accurately account for the total surface area when compared with BET data, so a surface roughness factor of about three is typically applied. Similarly, water will eventually penetrate the grain boundaries of multi-grained specimens, thereby increasing the effective surface area by at least a factor of five [1]. To avoid these problems Gray [1,6] performed BET on specimens that had been crushed, sieved, and washed to yield individual grains and to remove fines. Hanson et. al. [7] used fuel from the same section of the ATM-106 fuel used by Gray, but crushed the fuel and performed multiple sieving to remove fines without washing prior to performing BET. The BET results are $0.277 \mathrm{~m}^{2} \mathrm{~g}^{-1}$ [1], $0.102 \mathrm{~m}^{2}$ $\mathrm{g}^{-1}$ [6], $0.28 \mathrm{~m}^{2} \mathrm{~g}^{-1}$ [7] and $0.29 \mathrm{~m}^{2} \mathrm{~g}^{-1}$ [7]. With one notable exception, the surface area of the fuel from the same rod had excellent reproducibility.

Even if the initial surface area can be accurately and precisely determined, it is not known how the surface area changes as the fuel alters. In the case of static dissolution tests, StroesGascoyne et. al. [3] have reported that some grain boundary attack has occurred near the fuel pellet surface. The fragments exposed to unsaturated drip conditions have also exhibited grain boundary corrosion [4], which should increase the effective surface area exposed to water. Gray [1] has published numerous scanning electron microscopy (SEM) photographs of fuel grains and fragments after being subjected to flowthrough tests. In the case of fragments, behavior similar to that in Figure 1 is often observed. There is clearly a significant increase in the surface area as the grain boundaries corrode. For individual grains, the surface area appears to increase as a result of the formation of the "wormy" and pitted surfaces that result from the dissolution of the fuel.

As reported by Gray [1], one sample (corresponding to his specimen 15) had particle size distribution analyses performed and, with a surface roughness factor of three, had an initial specific surface area of $0.0858 \mathrm{~m}^{2} \mathrm{~g}^{-1}$. After being subjected to flowthrough dissolution where the cumulative release of uranium showed approximately $2 \%$ reaction, BET was performed and yielded a surface area of $0.444 \mathrm{~m}^{2} \mathrm{~g}^{-1}$, an increase of a factor of five. Yet, examination of the release rates shows a decrease over time. An examination of unreported data by Gray shows that one batch of ATM-103 fuel had an initial specific surface area of $0.074 \mathrm{~m}^{2} \mathrm{~g}^{-1}$. The fuel was then subjected to flowthrough tests at a $\mathrm{pH}$ of 4 and 6 , with cumulative reactions based on uranium releases of $73 \%$ and $66 \%$, respectively. BET on the remaining samples yielded specific surface areas of $25.7 \mathrm{~m}^{2} \mathrm{~g}^{-1}$ and $16 \mathrm{~m}^{2} \mathrm{~g}^{-1}$, respectively. In all cases, the surface area as measured by BET has increased significantly, verifying the observations seen in the SEM photographs. While it may be that the $\mathrm{Kr}$ gas used in BET testing is able to penetrate areas that 
water can not, thus giving an artificially high specific surface area, it is clear that dissolution rates obtained from static, drip, or flowthrough tests have not increased as the surface area has increased. In fact, the dissolution rates when normalized to a constant initial surface area typically decrease.

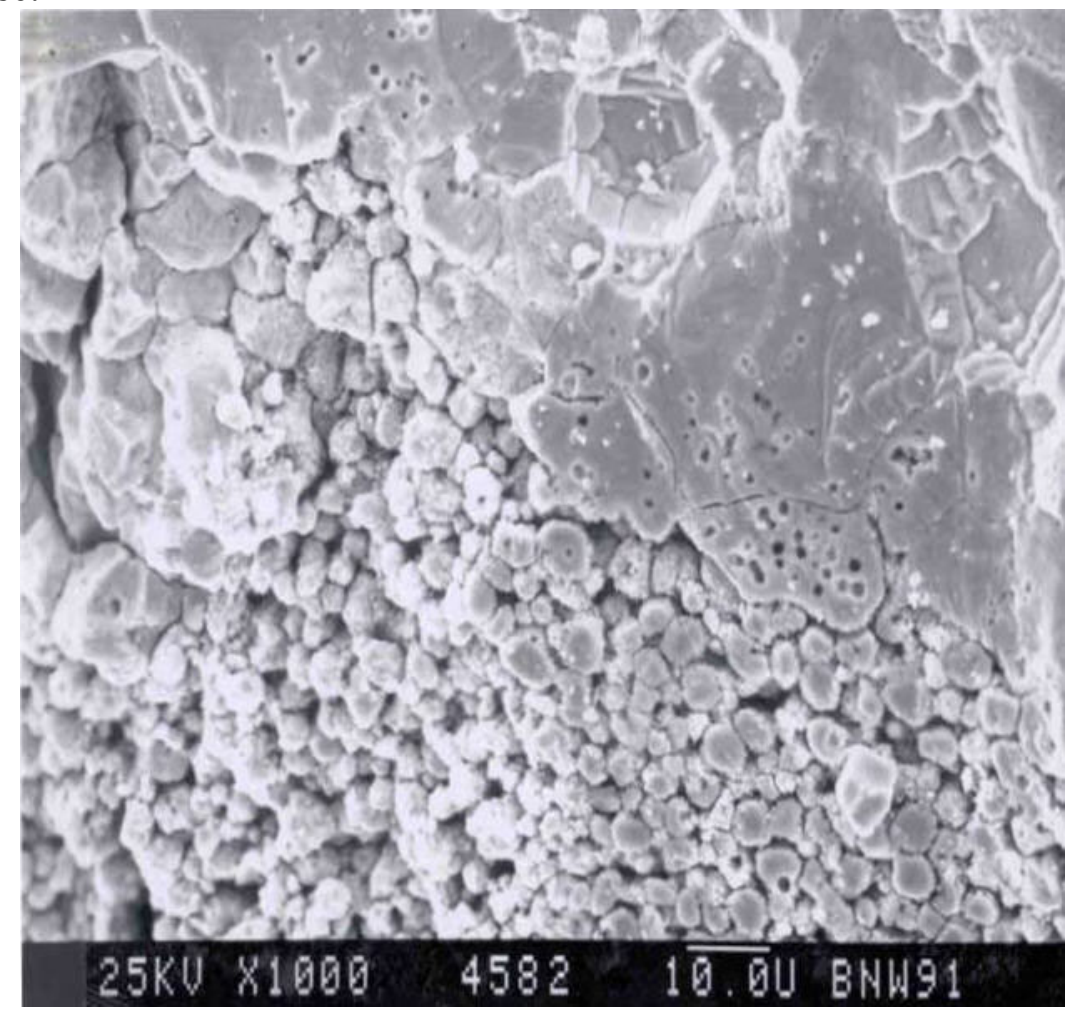

Figure 1. SEM photograph of a fuel fragment after flowthrough dissolution.

It is clear that some other phenomenon must exist that counters the effect of apparent increasing surface area. In his study on dissolution of natural uraninite crystals, Grandstaff [11] showed that the dissolution rate was greatly affected by the presence of non-uranium cations. By comparing the rate of dissolution for natural uraninite crystals containing differing concentrations of impurities, mostly $\mathrm{Th}$ and $\mathrm{Pb}$, he found that as the impurity concentration increased from $10 \%$ to $30 \%$, the dissolution rate decreased by more than a factor of 100 . The effect was most pronounced for Th, where the rate decreased by more than a factor of 10 as the $\mathrm{ThO}_{2}$ concentration increased from two to four percent. Grandstaff explains that the decrease could be the result of a slower rate of desorption of non-uranium cations at the surface of the fuel until those cations are either removed by reaction or by excavation of the surrounding uranium cations. The more non-uranium cations encountered, the slower the overall rate. A second factor is that $3+$ and $4+$ cations (such as the rare earth elements, $\mathrm{Zr}$, and $\mathrm{Pu}$ ) that won't exist in higher oxidation states will serve as effective negative charges making it more difficult for the nearest neighbor uranium ions to oxidize. The number of donor-acceptor sites available for the reduction of $\mathrm{O}_{2}$ at the fuel surface is thereby reduced, again slowing the dissolution rate.

\section{RESULTS}

The dissolution rates as normalized to the initial surface area for four different specimens are plotted in Figure 2. Included in the figure are the BET measured specific surface areas and 
the average normalized dissolution rate once an approximate steady state rate was achieved. It is clear that there is significant scatter in the data and that the two ATM-106 samples exhibit rates that differ by a factor of about 2.3. The initial specific surface areas for the two samples differ by a factor of about 2.6 , almost identical to the difference in rate. Since the fuel samples came 

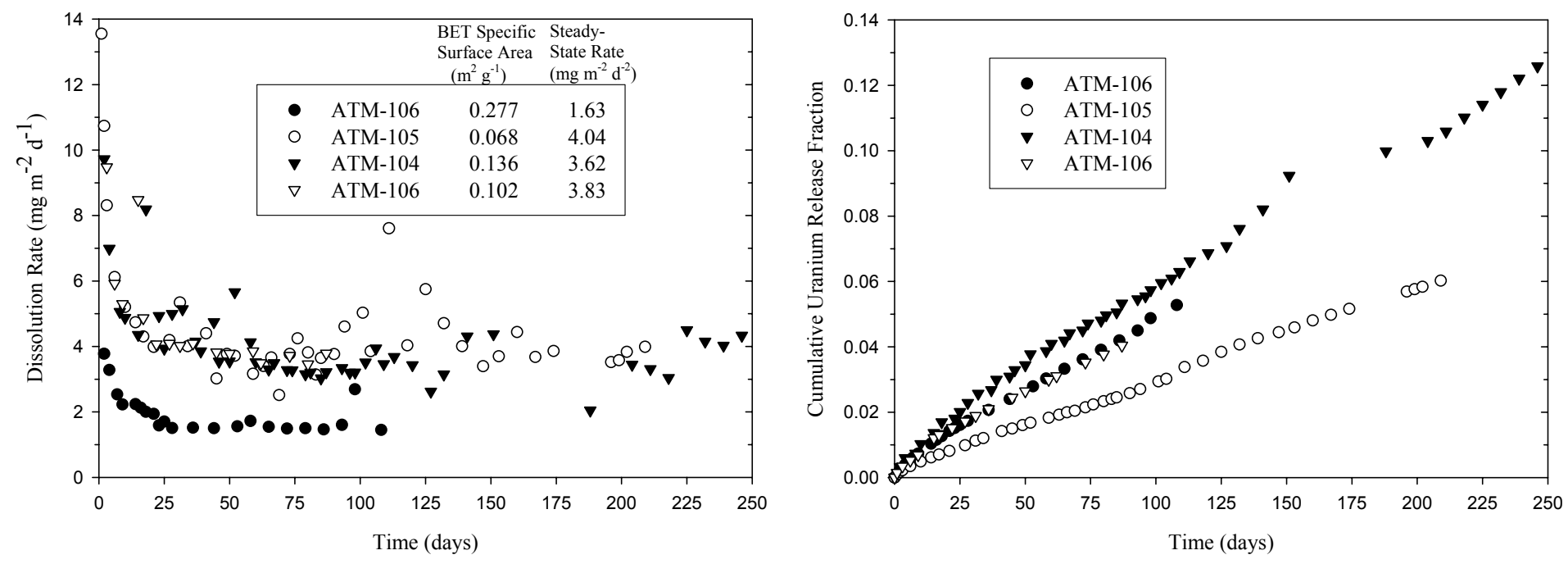

Figure 2. Dissolution rates of spent fuel in aerated water containing $2 \mathrm{E}-2 \mathrm{M}$ carbonate/bicarbonate, $\mathrm{pH}=8$, at $25^{\circ} \mathrm{C}$
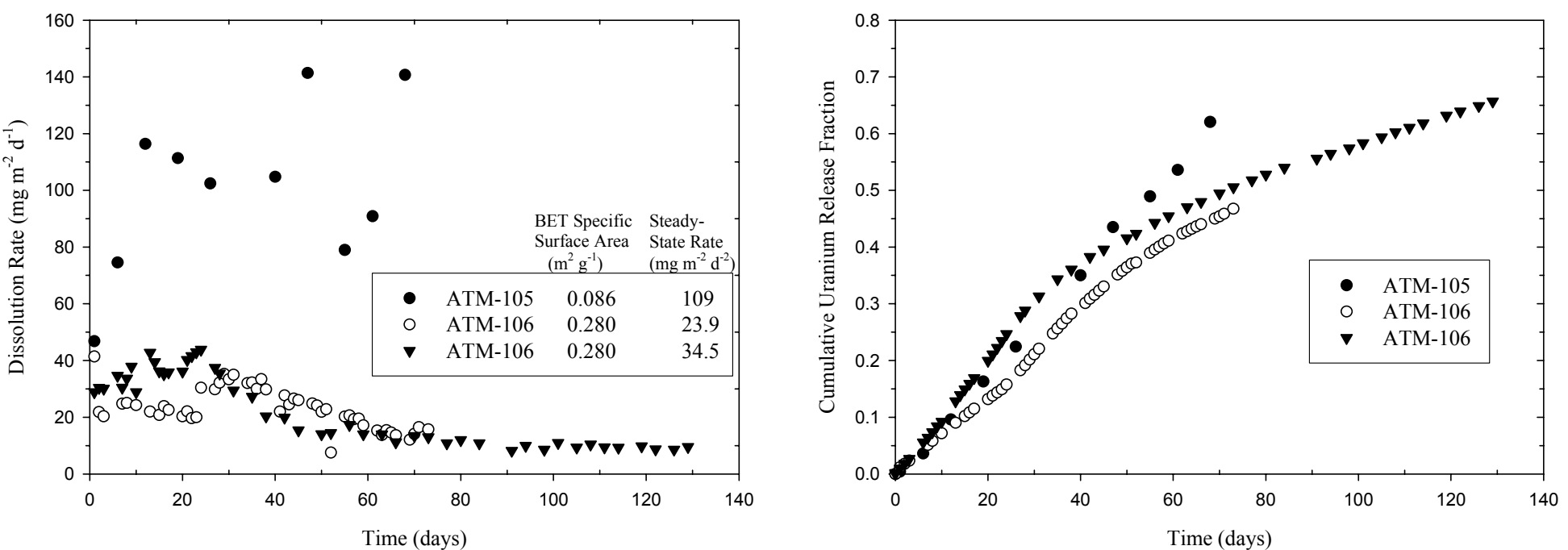

Figure 3. Dissolution rates of spent fuel in water with $\mathrm{HNO}_{3}$ added to make $\mathrm{pH}=3$ at $25^{\circ} \mathrm{C}$ 
from the same segment of the same fuel rod, we would expect the intrinsic dissolution rates to be the same. Indeed, when the cumulative release fractions based on uranium are plotted as a function of time, we see that the two samples are nearly identical. The ATM-104 fuel has a similar burnup [1] and its cumulative release curve would be nearly identical to the two ATM106 samples if the early initial release for the former is ignored. Based on the cumulative release curves, it appears that the intrinsic rate for dissolution of the two fuels is nearly the same. The ATM-105 fuel with a slightly lower burnup exhibits a smaller cumulative release, although the normalized rate is about that of the other samples.

It is tempting to simply assume that one of the BET measurements for ATM-106 was incorrect. However, in order to achieve the desired result, the larger surface area would have to decrease by about a factor of two. As noted earlier, however, two other analyses on similarly prepared ATM-106 fuel had specific surface areas similar to this larger value. It appears that the data and analyses are correct, but that normalizing to the surface area introduces a significant uncertainty.

A second comparison was made with samples tested at $\mathrm{pH} 3$ and $25^{\circ} \mathrm{C}$, as plotted in Figure 3. The original measurement was performed on ATM-105 fuel and exhibited a very large dissolution rate when normalized to the surface area. Two different tests on ATM-106 were performed. In the first, the feed solution was open to the atmosphere, but was not continuously sparged as were the other two samples. The normalized rate obtained is a factor of about 4.6 smaller than that of ATM-105. At first it was believed that the lack of oxygen from not sparging the solution was the cause of this smaller rate. The test was repeated with a fresh sample [7], but this time the feed solution was continuously stirred and sparged with $\mathrm{CO}_{2}$-free air, the same as the ATM-105 sample. The normalized rates for the two ATM-106 samples agree quite well, especially considering the potential oxygen deficiency of the first sample.

When all the samples are plotted as the cumulative release fraction as a function of time, the curves are all very similar as would be expected. It thus appears that by comparing cumulative release curves as opposed to rates normalized to the surface area, those samples that are expected to have similar rates do. In addition, it appears that dependencies on variables such as burnup become more pronounced when using the cumulative method.

It is important to stress that when comparing data, especially among different groups or between different sized samples (powders vs. fragments vs. pellets), that normalizing to the specific surface area may introduce significant uncertainty. The decrease in effective surface area caused by non-uranium cations at the specimen surface or by decreasing donor-acceptor sites for $\mathrm{O}_{2}$ reduction should apply to both spent fuel and SIMFUEL. In the case of $\mathrm{UO}_{2}$, the effective surface area should be unaffected by dissolution due to these phenomena. However, $\mathrm{UO}_{2}$ is a semiconductor and thus some areas will act as an anode and other areas as a cathode [12]. As the relative size of these local zones becomes increasingly small, then dissolution should still be directly proportional to the exposed surface area. If, on the other hand, corrosion results in preferential dissolution and the relative area acting as an anode decreases, then the dissolution rate should decrease for $\mathrm{UO}_{2}$ as well. Additional study of these mechanisms is warranted.

Finally, it is necessary to emphasize that even the cumulative method can not be used for direct comparison of samples with markedly different surface areas. That is, the fractional releases reported here are simply the amount of uranium released compared to the total uranium present initially, or a rate normalized to mass. Clearly, the significantly higher specific surface area of a powder relative to fragments or pellets will result in a higher total release. Future 
discussions of fuel dissolution should include detailed information on the type of sample used, the starting surface area, and both surface area normalized and cumulative release dissolution rates to allow an accurate comparison to be made.

\section{CONCLUSIONS}

A reexamination of data from flowthrough tests revealed that comparison of cumulative release curves eliminated some of the past discrepancies observed when dissolution rates are normalized to the starting surface area. Evidence in the form of BET analyses and SEM photographs was presented to show that the estimated surface area increases relatively rapidly after only a few percent reaction. Yet, even with an increasing surface area, the dissolution rates decrease when normalized to the fixed initial surface area. Arguments for these decreasing rates because of the non-uranium cations in the matrix and the accompanying decrease in donoracceptor sites for $\mathrm{O}_{2}$ reduction were presented. It is recommended that in the future, both surface area normalized rates and cumulative rates be examined when attempting to determine the parametric dependence of spent fuel dissolution.

\section{ACKNOWLEDGMENTS}

This work was performed in support of the Yucca Mountain Project and was funded through the Waste Form Department at Bechtel SAIC Corporation, LLC, the contractor for the Office of Civilian Radioactive Waste Management of the U.S. Department of Energy. This work was performed under the auspices of the U.S. Department of Energy by University of California, Lawrence Livermore National Laboratory under Contract W7405 -Eng-48.

\section{REFERENCES}

1. W.J. Gray and C.N. Wilson, Spent Fuel Dissolution Studies FY 1991 to 1994, PNL-10540, Pacific Northwest National Laboratory, December 1995.

2. S. Brunauer, P. Emmet, and E. Teller, J. Am. Chem. Soc. 60, 309(1938).

3. S. Stroes-Gascoyne, L.H. Johnson, J.C. Tait, J.L. McConnell, and R.J. Porth, in Scientific Basis for Nuclear Waste Management XX, edited by Walter J. Gray and Ines R. Triay, (Mater. Res. Soc. Proc. 465, Pittsburgh, PA, 1997) pp. 511-518.

4. R.J. Finch, E.C. Buck, P.A. Finn, and J.K. Bates, in Scientific Basis for Nuclear Waste Management XXII, edited by D.J. Wronkiewicz and L.H. Lee, (Mater. Res. Soc. Proc. 556, Warrendale, PA, 1999) pp. 431-438.

5. S. Röllin, K. Spahiu, and U.-B. Eklund, J. Nuc. Mat. 297, 231-243 (2001).

6. W.J. Gray, Spent Fuel Dissolution Rates as a Function of Burnup and Water Chemistry, PNNL-11895, Pacific Northwest National Laboratory, June 1998.

7. B.D. Hanson, J.I. Friese, and C.Z. Soderquist, presented at the 2004 MRS Spring Meeting, San Francisco, CA, 2004.

8. J.A. Serrano, J.P. Glatz, E.H. Toscano, J. Barrero, and D. Papaioannou, J. Nuc. Mat. 294, 339-343 (2001).

9. D.W. Shoesmith, J. Nuc. Mat. 282, 1-31 (2000).

10. V.M. Oversby, Uranium dioxide, SIMFUEL, and spent fuel dissolution rates- a review of published data, SKB Technical Report 99-22, Swedish Nuclear Fuel and Waste Management Co., 1999.

11. D.E. Grandstaff, Economic Geology, 71, 1493-1506 (1976).

12. F. Habashi and GA Thurston, Energia Nucleare, 14 (4) 238-244 (1967). 